\title{
Effects of Tunneling Current on STM Imaging Mechanism for Alkanethiol Self-assembled Monolayers on Au(111)
}

\author{
Abdulla Hel Al Mamun, Seung Bae Son, and Jae Ryang Hahn* \\ Department of Chemistry and Research Institute of Physics and Chemistry, Chonbuk National University, \\ Jeonju 561-756, Korea. *E-mail: jrhahn@jbnu.ac.kr \\ Received August 4, 2010, Accepted November 17, 2010
}

\begin{abstract}
We investigated the effects of tunneling current on scanning tunneling microscopy (STM) images of 1-octanethiol (OT) and 1-decanethiol (DT) self-assembled monolayers (SAMs). At a low tunneling current, the domain boundaries and ordered alkanethiol molecules were clearly resolved. As the tunneling current was increased at a constant bias voltage, however, the STM images showed disordered structures of the OT and DT SAMs. As the tunneling current was reduced back to low values, the ordered structures of the alkanethiol molecules reappeared. The reversibility of the process suggests that the sulfur head groups did not rearrange under any of the tunneling current conditions. On the basis of our observations, which are inconsistent with the standard model for STM imaging of molecules on metal surfaces, we consider the STM imaging mechanism in terms of a two-region tunneling junction model.
\end{abstract}

Key Words: 1-Octanethiol, 1-Decanethiol, Self-assembled monolayers, Au(111), Scanning tunneling microscopy (STM)

\section{Introduction}

Alkanethiol monolayers on $\mathrm{Au}(111)$ surfaces are the most extensively investigated self-assembled monolayers (SAMs) because they are model systems for understanding moleculesubstrate and molecule-molecule interactions as well as surface phase transitions. ${ }^{1}$ Many studies have described surface phase transitions, molecular adsorption sites, and the desorption mechanism for SAMs, thus providing a basis for exploiting SAMs in molecular devices, biosensors, chemical sensors, lubricators, and corrosion inhibitors. ${ }^{2-11}$ At the boundaries of chemistry, ${ }^{12}$ biology, ${ }^{13}$ and electronics, ${ }^{14}$ SAMs offer a versatile platform for nanotechnology. ${ }^{15}$

Molecular scale images reveal that alkanethiol SAMs form a $(\sqrt{3} \times \sqrt{3}) \mathrm{R} 30^{\circ}$ overlayer on $\mathrm{Au}(111)$ surfaces, which is further structured by a $c(4 \times 2)$ superstructure ${ }^{16-20}$ that is readily observed by scanning tunneling microscopy (STM) with high-impedance tunneling junctions. ${ }^{16,21-25}$ Although many reports have described alkanethiol SAMs in the last 20 years, a full understanding of this system is far from complete, which is mostly due to the lack of understanding of imaging mechanism. Characterization of the adsorption sites of the thiol groups on $\mathrm{Au}$ and the mechanism by which the SAM layers are imaged would assist development of next-generation applications of these SAMs. In general, the ordered structures of alkanethiol SAMs are obtained by STM imaging at very low tunneling currents (below 100 pA) and high bias voltages, possibly due to the low conductivity of the alkyl chains. The long alkyl chains of the thiols are frequently modeled as insulators, ${ }^{26}$ and previous work has assumed that the alkyl chains do not contribute to the tunneling current during STM imaging. ${ }^{22,27}$ These reports neglected the possible effects of the chain lengths on imaging mechanism when analyzing the STM images of SAMs. However, Weiss et al. proposed a model for the influence of the chain on electron tunneling in an explanation of the STM image contrast of SAM samples with mixed compositions. ${ }^{28}$ In their model, the conductance of the SAM films decreased much more slowly as the alkyl chain length increased than it did for an increase in air gap thickness. The decay constant of the air was larger $\left(0.23 \mathrm{~nm}^{-1}\right)$ than that of the SAMs $\left(0.12 \mathrm{~nm}^{-1}\right)$.

In the present work, we studied STM images of alkanethiol SAMs on $\mathrm{Au}(111)$ surfaces as a function of the STM tunneling current. These results characterized a trend that was opposite to the trend observed in molecular imaging of other systems on metal surfaces. To eliminate chemical-specific effects that could produce STM contrast, we chose two alkanethiols with different alkyl chain lengths so that the image contrast of these SAMs was due solely to alkyl chain length. The effects of alkyl chain length on STM imaging is discussed in terms of a tworegion tunneling junction model.

\section{Experimental}

The Au(111) surfaces were prepared either by vapor deposition of gold onto a freshly cleaved heated muscovite mica or onto a bead formed at the end of a Au wire. The OT ( $\geq 98.5 \%$, Aldrich) and DT (99\%, Aldrich) were used without further purification. The SAMs were formed by immersing the $\mathrm{Au}(111)$ surface into a distilled ethanol solution of alkanethiol (4 mM) in a stainless steel bath at $78{ }^{\circ} \mathrm{C}$ for 24 hours. After immersion, the samples were rinsed with ethanol to remove any weakly adsorbed species, followed by drying under nitrogen gas flow. All STM images reported here were obtained using a scanning tunneling microscope (SOLVER P47, NT-MDT) under ambient conditions. Image processing was performed by Nanotec WSxM software. An STM tip was mechanically cut from a $0.20 \mathrm{~mm}$ diameter platinum/iridium wire.

\section{Results and Discussion}

Figure 1(a) shows an STM topographical image of an OTSAM formed on the $\mathrm{Au}(111)$ surface. The high resolution image 

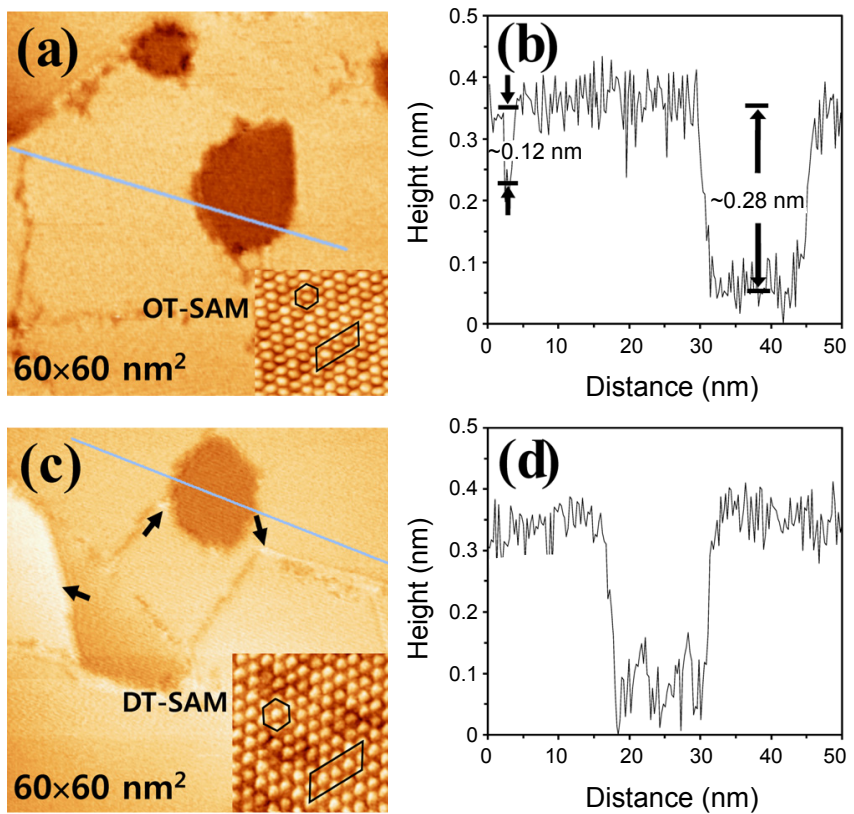

Figure 1. (a) STM topographical images of the OT-SAM on $\mathrm{Au}(111)$ obtained at a sample bias voltage of $600 \mathrm{mV}$ and a tunneling current of $150 \mathrm{pA}$. The inset shows the $(\sqrt{3} \times \sqrt{3}) \mathrm{R} 30^{\circ}$ hexagonal lattice pattern and its $(4 \times 2)$ superlattice structure. The scan area was $60 \times 60 \mathrm{~nm}^{2}$. (b) The cross-sectional cut shown in (a) shows the measured $2.8 \AA$ depth of the Au vacancy island and the measured $1.2 \AA$ depth of the domain boundary. (c) STM topographical images of DT-SAM on Au(111), obtained at a sample bias voltage of $1000 \mathrm{mV}$ and a tunneling current of $40 \mathrm{pA}$. The scan area was $60 \times 60 \mathrm{~nm}^{2}$. The inset shows a high resolution STM image of the $(\sqrt{3} \times \sqrt{3})$ R $30^{\circ}$ hexagonal lattice pattern and its $(4 \times 2)$ superlattice structure. (d) The cross-sectional cut shown in $(c)$

(inset) shows the $(\sqrt{3} \times \sqrt{3}) \mathrm{R} 30^{\circ}$ hexagonal pattern of OT-SAM and its $c(4 \times 2)$ superstructure. The $(\sqrt{3} \times \sqrt{3}) \mathrm{R} 30^{\circ}$ structure of the OT molecules was also visible within the Au vacancy islands, indicating that the Au vacancy islands were occupied by ordered OT. Analysis of the cross-sectional cut shown in Fig. 1(b) indicated that the measured depth, $2.8 \AA$, of the vacancy island was consistent with the Au step height. In addition to the domains and $\mathrm{Au}$ vacancy islands, the domain boundaries were an important feature of the STM topography of the OT-SAM. These boundaries appeared topographically lower (darker) by as much as $0.12 \mathrm{~nm}$, and no structure could be resolved within the domain boundaries. This thickness was far less than the physical height of the OT monolayer $(0.982 \mathrm{~nm}){ }^{29}$ Similar results were obtained from the DT-SAM. Figure 1(c) shows an STM topographical image of a DT-SAM, obtained at a sample bias voltage of 1000 $\mathrm{mV}$ and a tunneling current of $40 \mathrm{pA}$. In comparison with Fig. 1(a), Fig. 1(c) showed distinct bright features, as indicated by the arrows.

To further investigate the effects of the alkyl chain during STM imaging of the alkanethiol SAMs, a series of STM images of the DT-SAM were collected over a range of applied tunneling currents, from 40 to $500 \mathrm{pA}$ then back to $50 \mathrm{pA}$, at a fixed sample bias voltage of $1000 \mathrm{mV}$. At the initial tunneling current of $40 \mathrm{pA}$ (corresponding to a tunneling resistance of $25 \mathrm{G} \Omega$ ), ordered domain structures of the DT-SAM could be clearly
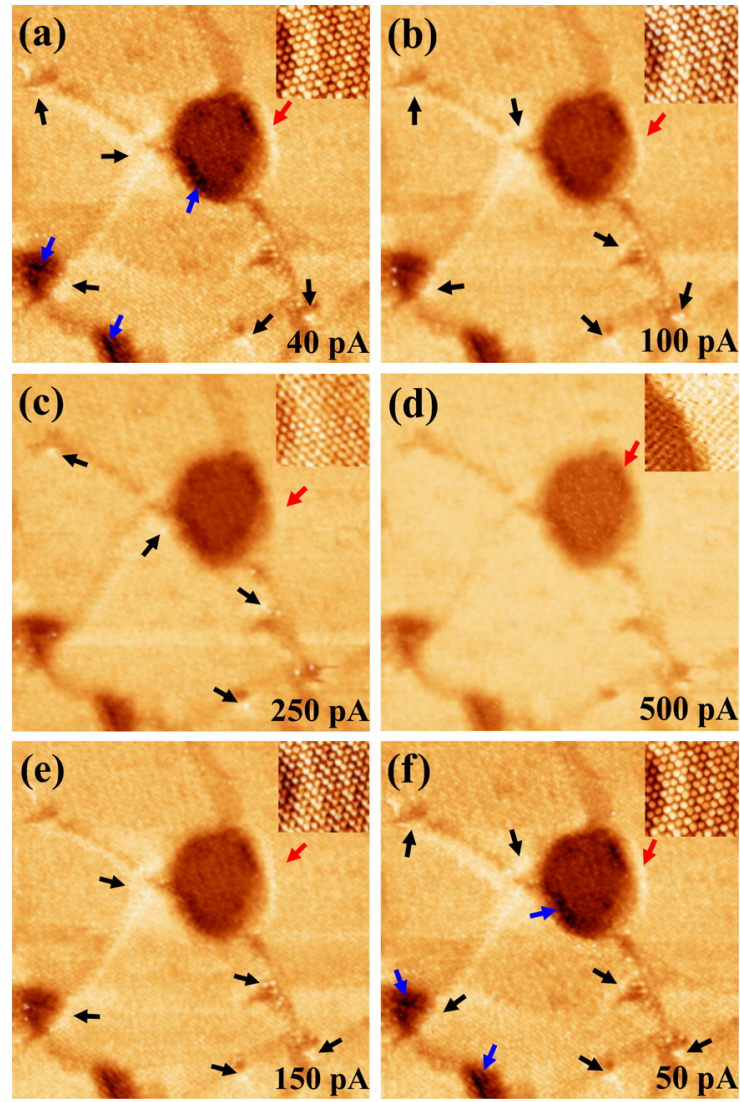

Figure 2. Sequential STM images of DT-SAM $\left(35 \times 35 \mathrm{~nm}^{2}\right)$ at a sample bias voltage of $1000 \mathrm{mV}$. The tunneling currents were (a) 40 $\mathrm{pA}$, (b) $100 \mathrm{pA}$, (c) $250 \mathrm{pA}$, (d) $500 \mathrm{pA}$, (e) $150 \mathrm{pA}$, and (f) $50 \mathrm{pA}$, respectively. The black arrows in the images indicate regions of closely packed molecules, blue arrows in Figs. 2(a) and 2(f) indicate more disordered regions, and red arrows indicate inset images of those regions.

observed in Fig. 2(a) without destruction of the monolayer. The black arrows in the images indicate regions of closely packed molecules, blue arrows in Figs. 2(a) and 2(f) indicate more disordered regions, and red arrows indicate inset images of those regions. As the tunneling current reached $100 \mathrm{pA}[10 \mathrm{G} \Omega$ tunneling resistance, shown in Fig. 2(b)], the domain structures of the DT monolayer changed dramatically and the imaging process became slightly disordered, although ordered atomic scale structures were still observed. A further increase in the tunneling current to $250 \mathrm{pA}$ (4 G $\Omega$ tunneling resistance), did not produce any pronounced changes in the domain image and bright features, except for slightly more destruction, as seen in Fig. 2(c). It is interesting that atomic resolution was achieved within some bright feature regions, but the structure of these bright regions differed from that of the surrounding regions. As the tunneling current increased to $500 \mathrm{pA}$ [as shown in Fig. 2(d), corresponding to a $2 \mathrm{G} \Omega$ tunneling resistance], the periodic structures, domain boundaries, and bright features gradually diminished until they could barely be discerned, and only gold vacancy islands were clearly visible. Restoring the tunneling current to $150 \mathrm{pA}$, as in Fig. 2(e), reproduced the bright features and destroyed-yet-ordered structures of the DT monolayer. As the 
tunneling current was reduced to $50 \mathrm{pA}$, as shown in Fig. 2(f), a well-ordered $(\sqrt{3} \times \sqrt{3}) \mathrm{R} 30^{\circ}$ hexagonal pattern on $\mathrm{Au}(111)$ was again observed. The above results suggest that even at high currents at which disordered imaging could be clearly observed [no ordered DT molecules, Fig. 2(d)], the DT molecules remained assembled on the gold substrate through Au-S bonds. Therefore, the sulfur head groups did not rearrange.

Features that were brighter than the surrounding features (indicated by several black and red arrows) were observed in-
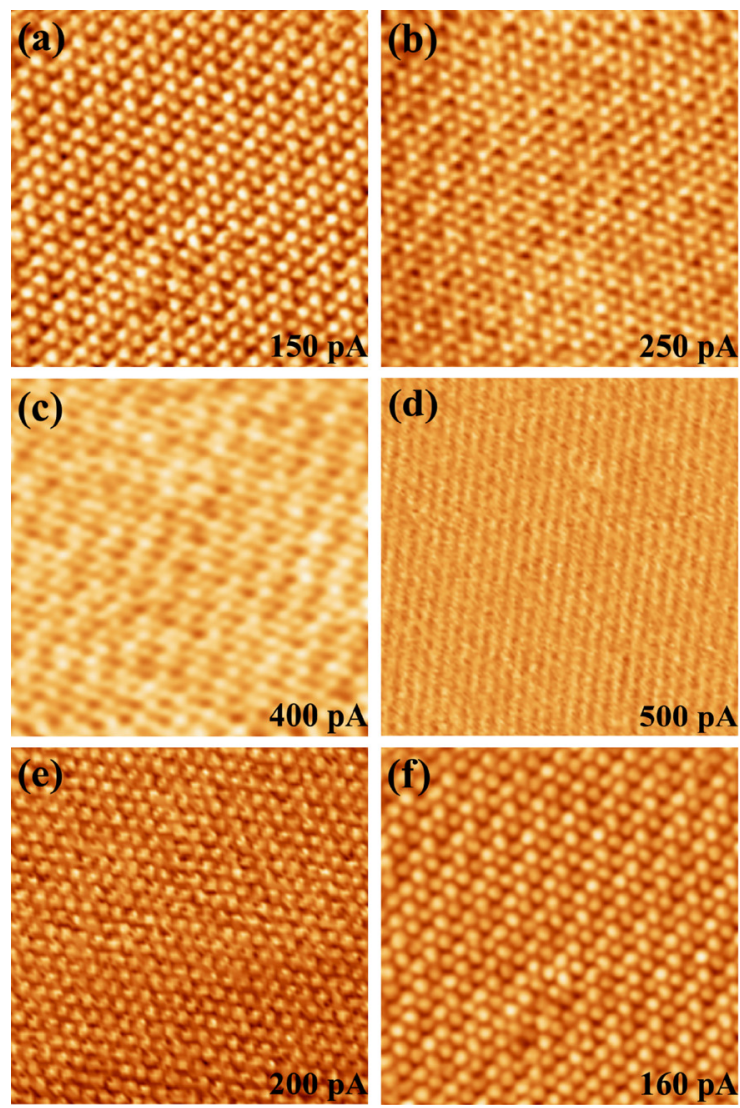

Figure 3. Sequential STM images of OT-SAM $\left(10 \times 10 \mathrm{~nm}^{2}\right)$ at a sample bias voltage of $600 \mathrm{mV}$. The tunneling current was set to (a) $150 \mathrm{pA}$, (b) $250 \mathrm{pA}$, (c) $400 \mathrm{pA}$, (d) $500 \mathrm{pA}$, (e) $200 \mathrm{pA}$, and (f) 160 $\mathrm{pA}$, respectively. dependent of the tunneling current. These bright features appeared to be more closely packed than the $(\sqrt{3} \times \sqrt{3})$ R $30^{\circ}$ structure. The packing order of the Au atoms may have changed near the gold vacancy islands, of the molecules may have assumed smaller tilt angles. Therefore, their physical height is higher than the area with $(\sqrt{3} \times \sqrt{3})$ R $30^{\circ}$ structure.

OT-SAM images of smaller areas at different tunneling currents are shown in Fig. 3. This image sequence shows the same trend as in Fig. 2. At 150 pA, the ordered structure was clearly observed. As the tunneling current was increased, the ordered structure began to blur, accompanied by structural changes. At $500 \mathrm{pA}$, it was almost impossible to distinguish the ordered structure. The line structures Fig. 3(d) may be due to a tip-induced effect. For example, alkanethiols might be pushed away from the STM tip because of small tip-sample gap space. However, when the tunneling current was reduced to $160 \mathrm{pA}$ [Fig. 3(f)], the ordered structure that reappeared was the same as that shown in Fig. 3(a). Figures 2 and 3 display an identical trend for both DT and OT as a function of tunneling current. In both cases, the hexagonal structure was reproducible throughout the imaging procedure. No permanent rearrangement of the sulfur head groups occurred under different tunneling currents. The changes in the images at different tunneling currents may have been a result of different imaging mechanisms for STM on the alkanethiol SAMs.

The tunneling current was determined by the total conductance between the tip and the sample surface. For an alkanethiol SAM on a $\mathrm{Au}(111)$ surface, the relevant sample surface is the Au surface. At very low tunneling currents, the tip is positioned above the surface of the SAM films. In this system, the tunnel junction was formed by two regions: the air gap and the alkanethiol monolayer (Fig. 4). Because of small decay constant of alkanethiols, their existence increases the probability of the electrons tunneling from the Au substrate to the tip. ${ }^{30}$ This indicated that the total conductance determined by the tunneling probability for a tunneling path Au-S $\rightarrow$ alkyl-chain $\rightarrow$ STM tip at low tunneling currents should exceed the conductance of the path through which the electrons tunnel directly from the $\mathrm{Au}$ substrate, i.e., Au $\rightarrow$ STM tip. Here, we considered the conductance of the region between the alkanethiol molecules to be lower than the conductance of the molecules themselves because this region did not have a bridge for electron transfer
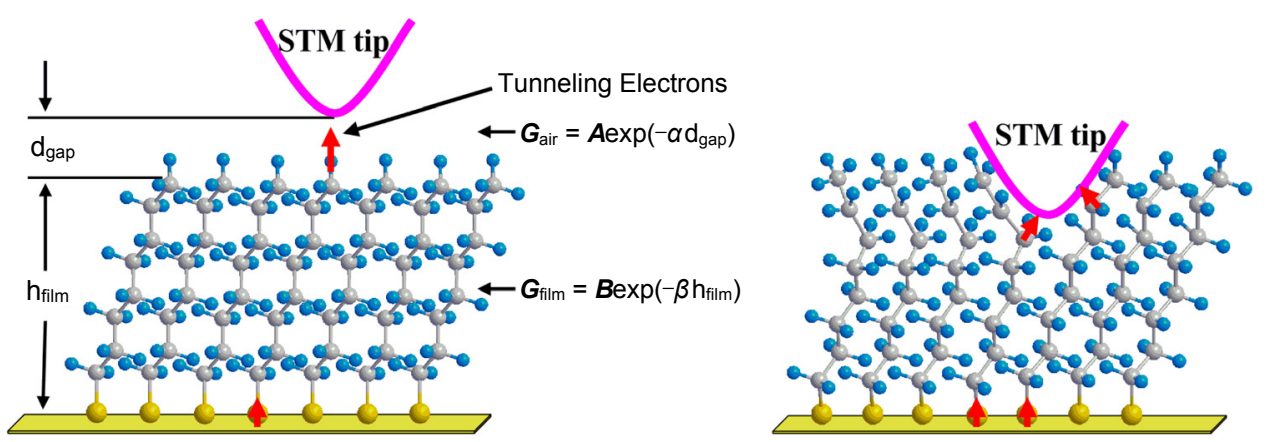

Figure 4. Models of the imaging process (a) at a low tunneling current and (b) at a high tunneling current. At a low tunneling current, the tunnel junction comprised two regions, air and the alkanethiol film. $\boldsymbol{G}_{\text {air }}$ and $\boldsymbol{G}_{\text {film }}$ are their corresponding conductances. $\boldsymbol{A}$ and $\boldsymbol{B}$ are the respective contact conductances. $\alpha$ and $\beta$ are the respective decay constants. $\mathrm{d}_{\text {gap }}$ and $\mathrm{h}_{\text {film }}$ are the thicknesses of the corresponding layers. The arrows indicate the possible paths of the tunneling current. At a high tunneling current, the tip penetrates the alkanethiol SAMs. 
of the alkanethiol molecules. Therefore, the tunneling electrons were expected to follow the path $\mathrm{Au}-\mathrm{S} \rightarrow$ alkyl-chain $\rightarrow \mathrm{STM}$ tip. Due to the large density of states of the Au atoms and the low barrier height among states, Au atoms can be a source of tunneling electrons. ${ }^{31}$ The alkanethiol molecules seem to be a part of tunneling current pathway. In this case, the STM image reflected the position of the sulfur head groups, as illustrated in Fig. 4(a), which produced a clear ordered structure at low tunneling current. As the tunneling current increased, the tip-substrate separation decreased.

Beyond a threshold tunneling current, ${ }^{28,30,32}$ the tip may penetrate the monolayer, as demonstrated in Fig. 4(b). Under such conditions, the tip may contact many molecules due to the relatively large size of the tip-apex compared to the alkanethiol molecules. Each molecule contacting the tip forms a pathway along which the tunneling current may flow from the Au substrate to the tip. The multiplicity of pathways blurs the image. Among these pathways, the molecule closest to the tip makes the largest contribution to the total current. Therefore, the ordered structure is still visible, although the observed structure should reflect the periodicity of the adsorbed surface rather than the actual atomic image. Therefore, the structure is disordered relative to the structure observed at low tunneling currents.

For a sufficiently small tip-substrate separation, the tunneling current through the pathway from the Au substrate directly to the tip may increase significantly such that it is no longer negligible. This could explain the disappearance of the ordered structure and grain boundaries at very high tunneling currents. Our model also provides a rationale for why the disordered regions were darker than the ordered regions at low tunneling currents, but were brighter as the tunneling current increased [as demonstrated by the blue arrows in Figs. 2(a) and 2(f)]. At the disordered regions (usually observed near gold vacancy islands or boundaries), the alkanethiol molecules may tilt at angles other than $30^{\circ}$, so that they are no longer parallel and may entangle with one another. This leads to a lower physical height in these regions than in the ordered regions and the regions appear darker. As the tunneling current increases beyond $40 \mathrm{pA}$ for DT, the tip may penetrate the alkanethiol film. As the tip approaches the disordered region where the alkanethiol molecules may be entangled, more molecules may contact the tip in this region than in the ordered region, which creates more channels through which the electrons may tunnel. These regions appear brighter than the ordered regions [Figs. 2(b) and 2(c)]. Further increases in the current $[500$ pA, Fig. 2(d)] result in even deeper penetration of the tip into the thiol monolayers, where the tip would be expected to contact a number of thiol molecules. This image should be bright over the whole scan area. However, due to contrast averaging in the STM software, the image displays an overall low contrast. As the tunneling current is subsequently reduced, the tip retracts. The imaging contrast reverses, the disordered regions become bright first [Fig. 2(e)], as shown in Figs. 2(b) and 2(c), and then return back to the state shown in Fig. 2(a) as the tunneling current is set back to $50 \mathrm{pA}$ [see Fig. 2(f)].

Imaging process of SAMs with tunneling electrons may be very different from that of flat-adsorbed molecules (e.g. hydroquinone $)^{33}$ on metal surface. The image of the flat-adsorbed molecules is determined by the convolution of electronic den- sity of states of the STM tip and molecular adsorbate. Assuming no resonant tunneling occurs through the molecule, the image contrast increases as the tip approaches the molecule on the surface (at a higher tunneling current and a lower sample bias voltages). The variation of electronic convolution is becoming more sensitive to the atomic structure. However the possible physical contacts between the STM tip and molecules may occur during SAM imaging at a closer proximity, which is resulting in a more complicated tunneling process.

\section{Conclusions}

We have investigated SAMs comprising both 1-octanethiol and 1-decanethiol on a $\mathrm{Au}(111)$ surface with atomic-scale resolution using STM, and we have proposed a model to explain the STM imaging mechanism for alkanethiol SAMs. Our model assumes that the conductance of the alkyl chains is larger than that of the air medium so that a channel consisting of $\mathrm{Au}-\mathrm{S} \rightarrow$ alkyl-chain $\rightarrow$ tip permits electron tunneling. This explains why the ordered structures of the SAMs, which reflect the positions of the sulfur atoms, are observed at low tunneling currents. The larger conductance of the alkyl chain reduces the height difference between the alkanethiol-adsorbed Au surface and the bare Au surface. Our results strongly suggest that the tunneling current through the alkyl chains plays an important role in STM imaging of SAMs.

Acknowledgments. This work was supported by the Korean government (NRF) (2010-0001638 and R01-2007-00020237-0). JRH was partially supported by the selection of research-oriented professor of Chonbuk National University in 2010 .

\section{References}

1. Vericat, C.; Vela, M. E.; Benitez, G. A.; Martin Gago, J. A.; Torrellels, X.; Salvarezza, R. C. J. Phys: Condens. Matter. 2006, 18, R867.

2. Allara, D. L. Biosens. Bioelectron. 1995, 10, 771.

3. Chen, J.; Reed, M. A.; Rawlett, A. M.; Tour, J. M. Science 1999, 286,1550

4. Hatzor, A.; Weiss, P. S. Science 2001, 291, 1019.

5. Smith, R. K.; Lewis, P. A.; Weiss, P. S. Prog. Surf. Sci. 2004, 75, 1.

6. Kumar, A.; Biebuyck, H. A.; Whitesides, G. M. Langmuir 1994, 10, 1498.

7. Mallouk, T. E.; Harrison, D. J. Interfacial Design and Chemical Sensing; American Chemical Society: Washington, DC, 1994.

8. Haussling, L.; Knoll, W.; Ringsdorf, H.; Schmitt, F. J.; Yang, J. L. Makromol. Chem., Macromol. Symp. 1991, 46, 145.

9. Allara, D. L.; Dunbar, T. D.; Weiss, P. S.; Bumm, L. A.; Cygan, M. T.; Tour, J. M.; Reinerth, W. A.; Yao, Y.; Kozaki, M.; Jones, L., II. Ann. N. Y. Acad. Sci. 1998, 852, 349.

10. Zamborini, F. P.; Crook, R. M. Langmuir 1998, 14, 3279.

11. Haussling, L.; Ringsdorf, H.; Schmitt, F. J.; Knoll, W. Langmuir 1991, 7, 1837.

12. Houseman, B. T.; Huh, J. H.; Kron, S. J.; Mrksich, M. Nat. Biotechnol. 2002, 20, 270.

13. Jonkheijm, P.; Weinrich, D.; Schroder, H.; Niemeyer, C. M.; Waldmann, H. Angew. Chem., Int. Ed. 2008, 47, 9618.

14. Van Hal, P. A.; Smits, E. C. P.; Geuns, T. C. T.; Akkerman, H. B.; De Brito, B. C.; Perissinotto, S.; Lanzani, G.; Kronemeijer, A. J.; 
Geskin, V.; Cornil, J. et al. Nat. Nanotechnol. 2008, 3, 749.

15. Madueno, R.; Raisanen, M. T.; Silien, C.; Buck, M. Nature 2008, $454,618$.

16. Camillone, N.; C. E. Chidsey, D.; Liu, G.; Scoles, G. J. Chem. Phys. 1993, 98, 3503.

17. Poirier, G. E.; Tarlov, M. J. Langmuir 1994, 10, 2853.

18. Delamarche, E.; Michel, B.; Gerber, C.; Anselmetti, D.; Güntherodt, H. J.; Wolf, H.; Ringsdorf, H. Langmuir 1994, 10, 2869.

19. Zhang, L.; Goddard, W. A., III; Jiang, S. J. Chem. Phys. 2002, 117, 7342.

20. Lüssem, B.; Müller-Meskamp, L.; Karthäuser, S.; Waser, R. Langmuir 2005, 21, 5256.

21. Poirier, G. E. Langmuir 1999, 15, 1167.

22. Poirier, G. E.; Pylant, E. D. Science 1996, 272, 1145.

23. Poirier, G. E. Chem. Rev. 1997, 97, 1117.

24. Fenter, P.; Eberhardt, A.; Eisenberger, P. Science 1994, 266, 1216.

25. Bucher, J. P.; Santesson, L.; Kern, K. Appl. Phys. A: Solids Surf. 1994, 59, 135.
26. Ulman, A. An Introduction to Ultrathin Organic Films; Academic Press: Boston, 1990.

27. Schönenberger, C.; Jorritsma, J.; Sondag-Huethorst, J. A. M.; Fokkink, L. G. J. J. Phys. Chem. 1995, 99, 3259.

28. Bumm, L. A.; Arnold, J. J.; Dunbar, T. D.; Allara, D. L.; Weiss, P. S. J. Phys. Chem. B 1999, 103, 8122.

29. Kim, J.; Uchida, H, Honda, N, Hashizume, N.; Hashimoto, Y, Kim, H.; Nishimura, K.; Inoue, M. Jpn. J. Appl. Phys. 2003, 42, 4770.

30. Salmeron, M.; Neubauer, G.; Folch, A.; Tomitori, M.; Ogletree, D. F.; Sautet, P. Langmuir 1993, 9, 3600.

31. Kittel, C. Introduction to Solid State Physics, 5th ed.; John Wiley: New York, 1976; p 154.

32. This current is different for different alkanethiol molecules; for 1-decanethiol and 1-octanethiol, these values are around $40 \mathrm{pA}$ and $150 \mathrm{pA}$, respectively. Therefore, shorter molecules require a larger tunneling current.

33. Inukai, J.; Wakisaka, M.; Itaya, K. Chem. Phys. Lett. 2004, 399, 373. 